\title{
Diagnóstico y tratamiento del dolor irruptivo oncológico: recomendaciones de consenso
}

\author{
Y. Escobar Álvarez ${ }^{1}$, A. Biete i Solà ${ }^{2}$, M. Camba Rodríguez ${ }^{3}$, R. Gálvez Mateos ${ }^{4}$, A. Mañas Rueda ${ }^{5}$, \\ C.A. Rodríguez Sánchez ${ }^{6}$, D. Rodríguez $\mathrm{Mesa}^{7}$ y A. Tuca i Rodríguez ${ }^{2}$ \\ ${ }^{1}$ Hospital Universitario Gregorio Marañón. Madrid. ${ }^{2}$ Hospital Clínic i Provincial. Universidad de \\ Barcelona. Barcelona. ${ }^{3}$ Hospital Arquitecto Marcide. El Ferrol, La Coruña. ${ }^{4}$ Hospital Universitario Virgen \\ de las Nieves. Granada. ${ }^{5}$ Hospital Universitario La Paz. Madrid. ${ }^{6}$ Hospital Universitario. Salamanca. \\ ${ }^{7}$ Hospital Universitari Sant Joan. Reus, Tarragona
}

Escobar Álvarez Y, Biete i Solà A, Camba Rodríguez M, Gálvez Mateos R, Mañas Rueda A, Rodríguez Sánchez CA, Rodríguez Mesa D, Tuca i Rodríguez A. Diagnóstico y tratamiento del dolor irruptivo oncológico: recomendaciones de consenso. Rev Soc Esp Dolor 2014; 21(2): 97-105.

\begin{abstract}
Introduction and objectives: Breakthrough cancer pain (BTcP) is an acute exacerbation of baseline pain. The clinicians involved in its management have different diagnostic and therapeutic criteria.

In order to facilitate decision making in usual clinical practice, 8 reference experts from 4 scientific associations involved in the management of patients with cancer pain have developed this Consensus Document.

Methods: After an initial search on the most relevant publications in BTcP literature, a set of preliminary recommendations were established. A working meeting was subsequently held with the experts, following the Metaplan ${ }^{\circledR}$ methodology -a structured brainstorming technique- that produced a first version of the Consensus Document which, after several review rounds, was validated by all the participants. Every statement and recommendation was sorted according to its degree of recommendation, following the categories in the SIGN (Scottish Intercollegiate Guidelines Network) system.

Results: The management of $\mathrm{BTcP}$ requires a full anamnesis, both of BTcP itself and of baseline pain, a physical examination and the supplementary tests that are deemed necessary.

The drugs of choice for the treatment of BTcP must be those with a potent and rapid analgesic effect a short duration, minimal side effects and easy administration. Transmucosal fentanyl is
\end{abstract}

Recibido: 15-12-12.

Aceptado: 01-03-13. currently the active ingredient most fitting to the analgesic needs of $\mathrm{BTCP}$, regardless of the major opioid used for control of the baseline pain.

Conclusion: This Consensus can be a very useful tool to improve the quality of life in cancer patients, because it guides the clinician towards a better diagnose and treatment of BTcP.

Key words: Breakthrough pain. Pain. Episodic pain. Cancer.

\section{RESUMEN}

Introducción y objetivos: el dolor irruptivo oncológico (DIO) es una exacerbación aguda del dolor que presenta diferentes criterios diagnósticos y de tratamiento por parte de los distintos especialistas implicados en su manejo.

Para facilitar la toma de decisiones en la práctica clínica habitual, ocho especialistas de referencia de cuatro sociedades científicas implicadas en el manejo del paciente oncológico han diseñado este documento.

Métodos: tras una búsqueda bibliográfica en las publicaciones más relevantes sobre DIO se establecieron las recomendaciones preliminares. El grupo de expertos realizó una reunión de trabajo siguiendo la metodología Metaplan ${ }^{\circledR}$ en la que se debatieron las recomendaciones que incorporar al documento. Cada una de las afirmaciones y recomendaciones fueron clasificadas según su grado de recomendación, atendiendo a las categorías del sistema SIGN (Scottish Intercollegiate Guidelines Network).

Resultados: el manejo del DIO requiere una anamnesis completa tanto del DIO como del dolor basal y una exploración física del paciente asociada a pruebas complementarias cuando sean precisas.

Los fármacos de elección para el tratamiento del DIO deben ser aquellos que muestren una analgesia potente, con rápido inicio, de efectos secundarios mínimos y de fácil administración. El fentanilo administrado por vía transmucosa es actualmente el principio activo más adecuado a las necesidades analgésicas del dolor irruptivo, con independencia del opioide mayor utilizado para el control del dolor basal. 
Conclusión: este consenso puede ser una herramienta útil para la mejora de la calidad de vida del paciente con cáncer, ya que permite un mejor diagnóstico y tratamiento del DIO.

Palabras clave: Dolor irruptivo. Dolor. Dolor episódico. Cáncer.

\section{INTRODUCCIÓN}

El dolor irruptivo oncológico (DIO) es un tipo de dolor asociado a procesos tumorales que se caracteriza por su elevada complejidad, a la cual contribuyen varios factores.

En primer lugar, ha sido definido de diversas maneras por distintos autores, lo cual es una muestra de la dificultad conceptual que implica, aunque hay algunos hechos que se aceptan de forma general, como que el DIO se presenta en pacientes con patología tumoral $(1,2)$ y que se acompaña de elevados niveles de depresión y ansiedad (2).

Por otro lado, su verdadera prevalencia no se conoce bien, pues varía notablemente en los diferentes estudios y series publicados; en general, se puede decir que su prevalencia es alta, incluso en los estadios tempranos de la enfermedad (3). Un reciente estudio de Mercadante y cols. (4) ha mostrado que algunos factores influyen en su aparición, como el estadio tumoral, las características del propio paciente y el tratamiento utilizado; en su serie, la prevalencia alcanza casi un $90 \%$.

Otra dificultad añadida es el hecho de que diferentes especialidades médicas están implicadas en su diagnóstico $\mathrm{y}$ tratamiento, lo cual puede dificultar la homogeneidad en su manejo.

Finalmente, se sabe que el DIO es un factor que limita la calidad de vida del paciente, implica una insatisfacción terapéutica y supone un elevado consumo de recursos sanitarios $(2,5,6)$.

No obstante, a pesar de todo ese conocimiento acumulado, es una realidad que en la actualidad el DIO está infradiagnosticado e infratratado.

Respecto a las opciones terapéuticas, sabemos que las características que definen el DIO -rápida instauración, elevada intensidad y breve duración- no encajan en el modelo de acción de los opioides tradicionales de acción rápida, que tardan unos 30 minutos en alcanzar la analgesia y su efecto 4 horas o más; es preciso disponer de fármacos que se ajusten al patrón del DIO, como es el caso de los fentanilos administrados por vía transmucosa $(7,8)$.

De la percepción de la necesidad de homogeneizar los conceptos básicos, los criterios diagnósticos y el tratamiento del DIO surge este consenso entre distintas especialidades, cuya finalidad es abrir una vía de estudio y desarrollo posterior que optimice todos esos aspectos.

\section{METODOLOGÍA}

Se estableció en España un grupo de trabajo multidisciplinar formado por ocho especialistas de referencia de diferentes ámbitos relacionados con el manejo del paciente oncológico: 2 especialistas de oncología médica, 2 de oncología radioterápica, 2 miembros de unidades de tratamiento del dolor y 2 miembros de unidades de cuidados paliativos (Tabla I). La metodología de trabajo que se estableció siguió diferentes fases y su secuencia se detalla en la figura 1.

TABLA I. EQUIPO MULTIDISCIPLINAR DE EXPERTOS QUE HAN PARTICIPADO EN LA ELABORACIÓN DEL CONSENSO

\begin{tabular}{lc}
\hline \multicolumn{1}{c}{$\begin{array}{c}\text { Miembro del grupo de } \\
\text { consenso }\end{array}$} & $\begin{array}{c}\text { Especialidad médica } \\
\text { representada }\end{array}$ \\
\hline $\begin{array}{l}\text { Dulce Rodríguez Mesa } \\
\text { Albert Tuca i Rodríguez } \\
\text { Manuel Camba Rodríguez }\end{array}$ & Cuidados paliativos \\
Rafael Gálvez Mateos \\
Yolanda Escobar Álvarez \\
$\begin{array}{l}\text { César A. Rodríguez Sánchez } \\
\text { Albert Biete i Solà }\end{array}$ & Unidad del dolor \\
Ana Mañas Rueda & Oncología médica \\
\hline
\end{tabular}

Recomendaciones iniciales $\downarrow$

Reunión de consenso

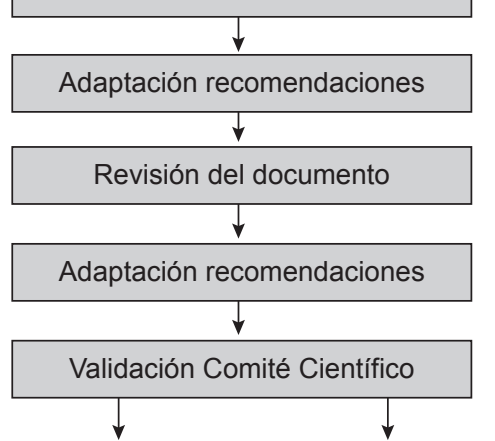

Solicitud de avales
Difusión y publicaciones
Fig. 1. Fases para la elaboración del documento de consenso.

En primer lugar se realizó una búsqueda bibliográfica dirigida que tuvo como objetivo recoger las publicaciones más relevantes hasta la fecha acerca del DIO.

Tras la lectura y análisis detallado de los artículos, se establecieron unas recomendaciones preliminares relativas al diagnóstico y tratamiento del dolor irruptivo, así como sobre otros aspectos generales y conceptuales. 
Tales recomendaciones fueron debatidas en una primera reunión presencial (reunión de consenso) del grupo de expertos en la que se empleó la metodología Metaplan ${ }^{\circledR}(9)$. Se trata de una técnica de brainstorming estructurado que utiliza diversos recursos participativos para la obtención de un conocimiento ordenado generado por un grupo de expertos; por lo general, un moderador experto y externo al grupo lanza a los asistentes unas preguntas previamente establecidas y sus respuestas son leídas posteriormente por dicho moderador y debatidas por el grupo. Las ventajas de esta metodología son las siguientes: permite una participación equitativa de todos los asistentes, así como la identificación de puntos de consenso y disenso, y permite tratar los temas de interés de forma eficiente y en un tiempo limitado.

Tras los comentarios y sugerencias de los participantes en dicha reunión, se modificaron las recomendaciones previas y se elaboró una primera versión del documento de consenso que fue enviado a los expertos para su consideración y validación. Una vez obtenido el documento final, cada una de las afirmaciones y recomendaciones fueron clasificadas según su grado de recomendación, atendiendo a las categorías del sistema SIGN (Scottish Intercollegiate Guidelines Network) (10) (Tabla II). Por último, el documento final de recomendaciones de consenso fue aprobado por todos los participantes.

\section{RECOMENDACIONES PARA EL DIAGNÓSTICO Y TRATAMIENTO DEL DOLOR IRRUPTIVO}

Las recomendaciones se han establecido sobre tres aspectos del DIO: conceptos generales, diagnóstico y tratamiento.

\section{Conceptos generales sobre el DIO}

\section{Dolor de base estabilizado}

- R.1: se considera un dolor de base estabilizado aquel dolor crónico que presenta el paciente cuya intensidad no varía en las últimas 48 horas* ni precisa una variación en la dosis de analgésico para controlarlo en el mismo periodo (11-13). Grado de recomendación: $D$ y $\sqrt{ }$.

\section{Definición del dolor irruptivo oncológico}

- R.2: se define el DIO como una exacerbación aguda del dolor de rápida aparición, corta duración y moderada a elevada intensidad que sufre el paciente cuando este presenta un dolor basal estabilizado y controlado con opioides (11-14). Grado de recomendación: D.

\section{Consideraciones generales del dolor irruptivo oncológico}

- R.3: se ha demostrado que el DIO disminuye la calidad de vida del paciente, por lo que es imprescindible su correcto diagnóstico y tratamiento (2). Grado de recomendación: $D$.

- R.4: el DIO es un síntoma que puede aparecer en cualquier momento del curso evolutivo de la enfermedad oncológica y que debe ser tratado y controlado tan pronto como aparezca. Grado de recomendación: D (tiene que haber una referencia pero no se ha transcrito, suponemos).

TABLA II. CATEGORÍAS DE GRADOS DE RECOMENDACIÓN SEGÚN EL SISTEMA SIGN (10)

$$
\text { Grados de recomendación }
$$

Al menos un metaanálisis, revisión sistemática o ensayo clínico clasificado como 1++ y directamente aplicable a

$A$ la población diana de la guía; o un volumen de evidencia científica compuesto por estudios clasificados como $1+$ y con gran consistencia entre ellos

Un volumen de evidencia científica compuesta por estudios clasificados como $2++$, directamente aplicable a la

$B$ población diana de la guía y que demuestran gran consistencia entre ellos; o evidencia científica extrapolada desde estudios clasificados como $1++$ o $1+$

Un volumen de evidencia científica compuesta por estudios clasificados como $2+$ directamente aplicables a la

$C$ población diana de la guía y que demuestran gran consistencia entre ellos; o evidencia científica extrapolada desde estudios clasificados como $2++$

D Evidencia científica de nivel 3 o 4; o evidencia científica extrapolada desde estudios clasificados como 2+ Buena práctica clínica

$\sqrt{ } \quad$ Práctica recomendada, basada en la experiencia clínica y el consenso del grupo de expertos

"La especificación "en las últimas 48 horas" es un aspecto de la recomendación propia de este grupo de expertos, por lo que se le otorga el nivel de recomendación $\sqrt{ }$. 
- R.5: un inadecuado manejo del DIO implica un mayor consumo de recursos sanitarios (5). Grado de recomendación: $D$.

\section{Clasificación del dolor irruptivo oncológico}

- R.6: para poder evaluar correctamente el DIO se recomienda clasificarlo según su etiología, su fisiopatología y los factores desencadenantes $(7,13,15)$. Grado de recomendación: $D$.

- R.7: según su etiología, el DIO puede ser causado por el propio tumor, por pruebas diagnósticas y por el tratamiento antitumoral o bien por procesos simultáneos al cáncer (aplastamiento vertebral, herpes zóster...) $(7,13)$. Grado de recomendación: $D$.

- R.8: según su fisiopatología, el DIO se puede clasificar en $(7,16)$ :

- Nociceptivo: si está relacionado con una activación de los nociceptores presentes en los distintos tejidos/órganos debido a daños tisulares (somático o visceral).

- Neuropático: si es debido a una lesión nerviosa periférica o central de la transmisión somatosensorial.

- Mixto: si el dolor presenta ambos tipos de características. Grado de recomendación: D.

- R.9: según los factores que lo desencadenan, el DIO se clasifica en (13):

- Dolor incidental: es el que aparece relacionado con alguna acción; a su vez se divide en:

- Volitivo, si se desencadena por una acción voluntaria (p. ej. deambulación, comer...).

- No volitivo, si se desencadena por una acción involuntaria (p. ej. tos, deglución...).

- Procedimental, relacionado con maniobras realizadas por o sobre el paciente (p. ej. cura de una úlcera, máscara para radioterapia).

- Dolor espontáneo o idiopático: hace referencia al que aparece sin un precipitante claro. Grado de recomendación: $D$.

- R.10: el dolor por fallo de final de dosis hace referencia al que aparece antes de la siguiente dosis de un analgésico pautado de forma regular para el dolor basal; se relaciona con un inadecuado control de dicho dolor crónico, por lo que no se considera un DIO y se aconseja excluirlo de la clasificación del mismo. Su control depende de un incremento de la dosis o una disminución del intervalo entre las dosis del opioide basal (12). Grado de recomendación: D.

- R.11: siempre que sea posible, es importante identificar y actuar sobre el agente causal del dolor irruptivo y hacerlo, preferentemente, de forma preventiva. No obstante, es imprescindible mantener al paciente con analgésicos adecuados para controlar el dolor basal y hacer un seguimiento estrecho para aumentar o disminuir el tratamiento analgésico en caso de variaciones (13). Grado de recomendación: $D$.

- R.12: es importante que el facultativo tenga conocimiento de la gran variabilidad de características que, según la literatura, presenta el DIO $(13,16)$ : Grado de recomendación: $D$.

- Tiempo hasta el pico de máxima intensidad: media de 3-5 minutos, con un rango de 10 segundos a 180 minutos.

- Intensidad: moderada a elevada/insoportable.

- Duración: media de 15-30 minutos, con un rango de 1 segundo a más de 240 minutos.

- Número de episodios al día: media de 1 a 4, con un rango de 1 a 14.

- Tipo incidental: media de 55-60 \% de los casos.

- Tipo incidental volitivo: media de 50-60\%.

- R.13: el DIO presenta una gran variabilidad interindividual, lo que conlleva la necesidad de adaptar el tratamiento a cada paciente $(13,16)$. Grado de recomendación: $D$.

- R.14: el facultativo debe tener en cuenta también la gran heterogeneidad intraindividual que presenta el DIO. En un mismo paciente puede seguir patrones diferentes en cuanto a tiempo de inicio, intensidad o duración; existe, además, heterogeneidad asociada al factor desencadenante en un mismo paciente $(13,16)$. Grado de recomendación: $D$.

\section{Diagnóstico del DIO}

- R.15: para poder realizar el diagnóstico es necesaria una anamnesis completa tanto del DIO como del dolor basal del paciente, una exploración física centrada en el dolor y pruebas complementarias cuando sean precisas $(7,13,16)$. Grado de recomendación: $D$.

- R.16: para realizar la anamnesis del DIO se recomienda, como mínimo, la valoración del número de episodios, la variedad fisiopatológica y las características del dolor (tiempo de inicio, duración, intensidad, frecuencia, localización e irradiación), los factores tanto desencadenantes como calmantes y la medicación utilizada hasta ese momento. Las evaluaciones deben realizarse siempre antes del inicio del tratamiento y posteriormente a intervalos frecuentes hasta lograr un adecuado control del dolor $(7,13,16)$. Grado de recomendación: $D$.

- R.17A: la anamnesis inicial del paciente debería incluir un diagnóstico diferencial de su dolor. Para ello, se formularán al paciente las preguntas correspondientes al algoritmo de Davies (Fig. 2) a fin de determinar si el paciente presenta o no DIO (13). Grado de recomendación: $D$. 


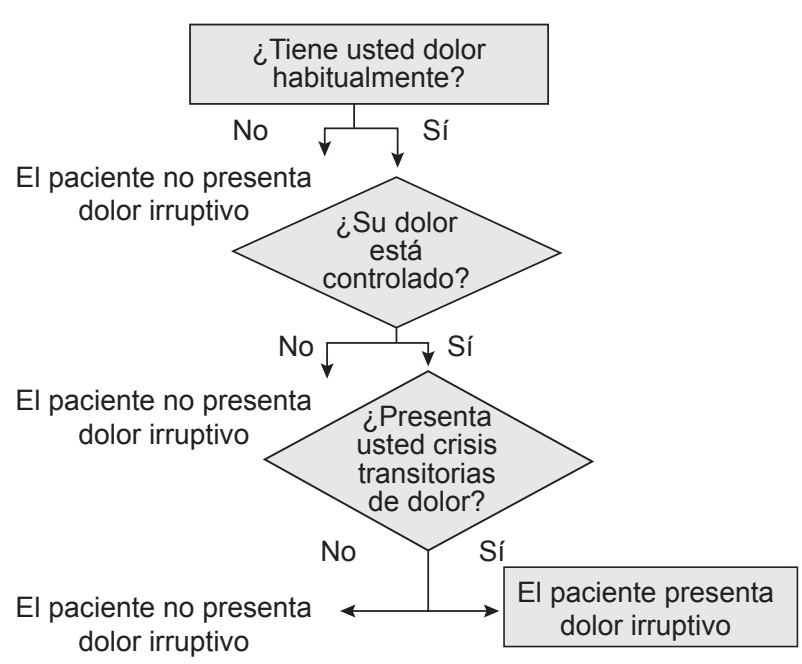

Fig. 2. Algoritmo de Davies modificado.

- R.17B: posteriormente, una vez diagnosticado el DIO, se llevará a cabo una anamnesis específica que incluirá las siguientes preguntas (13): Grado de recomendación: $D$.

- Las crisis, ¿son similares o diferentes a su dolor habitual? ¿Son iguales todas las crisis entre sí?

- ¿Cuántas crisis de dolor presenta al día? ¿Y a la semana?

- En una escala del 0 al 10, siendo 0 la ausencia de dolor y 10 el máximo dolor que se imagina que puede sufrir, ¿cómo puntuaría el dolor que presenta en cada crisis?

- ¿Cuánto tiempo pasa desde que se inicia el dolor hasta que llega a su máxima intensidad, segundos o minutos?

- ¿Cuánto tiempo dura cada crisis (minutos, horas)?

- Describa en qué lugar nota el dolor. ¿A qué se parece la sensación? ¿Hacia dónde se irradia?

- Cuando tiene la crisis, ¿está usted en movimiento o realizando alguna actividad, o bien aparece espontáneamente o justo antes de que le corresponda la siguiente dosis de analgésico para su dolor crónico?

- ¿Qué repercusión tiene este dolor en su vida diaria?

- ¿Le permite descansar por la noche? ¿Le impide hacer aquello que desea o necesita?

- ¿Hay alguna cosa que usted evita hacer para impedir que aparezca el dolor?

- ¿Qué tipo de medidas utiliza para aliviarlo? ¿Le son efectivas?

- ¿Ha dejado de tomar algún fármaco para el dolor por sus efectos secundarios? ¿Lo retiró por su toxicidad?

- ¿Qué fármacos utiliza para mejorar su dolor? ¿A qué dosis? ¿Son efectivos?
- R.18: la anamnesis del dolor, tanto al inicio como en los seguimientos, debe ser registrada en la historia clínica del paciente; el profesional sanitario debería anotar en ella, al menos, la presencia de DIO, el número de episodios, su duración e intensidad. Se registrará también la respuesta a los analgésicos de rescate utilizados previamente y la toxicidad de los mismos, la repercusión en la calidad de vida (sueño, ansiedad, depresión) y cualquier otra variable relevante planteada durante la anamnesis del paciente (16). Grado de recomendación: $D$.

- R.19: para facilitar el seguimiento, es útil que los pacientes anoten sus crisis de DIO para informar a su médico en cada visita (16). Grado de recomendación: $D$.

\section{Tratamiento del DIO}

\section{Definiciones de LAO, SAO y ROO}

- R.20: long acting opioids (LAO): formulaciones de opioides de liberación controlada (normalmente administrados por vía oral o transdérmica, si bien hay otras vías de administración) con una duración del efecto analgésico de 8 a 72 horas y un lento inicio de acción.

- Short acting opioids (SAO): formulaciones de opioides de liberación normal o rápida (normalmente administrados por vía oral, si bien hay otras vías de administración) con una duración del efecto analgésico de 4 a 6 horas y un inicio de acción a los 30-40 minutos.

- Rapid onset opioids (ROO): formulaciones de opioides de liberación inmediata (p. ej. las formulaciones de fentanilo) con una duración del efecto analgésico de 1 a 2 horas y un inicio de acción a los 3-15 minutos. Normalmente se administran por vía transmucosa (oral o nasal) (8). Grado de recomendación: D.

- R.21: el tratamiento analgésico del DIO es específico y no sustituye al tratamiento del dolor basal; se deben prescribir opioides para el DIO como complemento al tratamiento con opioides utilizados para controlar el dolor basal $(8,12,13,16,17)$. Grado de recomendación: $D$.

- R.22: el tratamiento del paciente con DIO debe ser integral, englobando la prevención y medidas terapéuticas analgésicas y coanalgésicas, tanto farmacológicas como no farmacológicas $(8,12,13,16,17)$. Grado de recomendación: $D$.

- R.23: el tratamiento analgésico del DIO con opioides debe ir acompañado, desde el inicio, de un tratamiento para la prevención de sus efectos secundarios (náuseas, vómitos, estreñimiento); si no es posible 
realizar una prevención de los mismos, el objetivo de la terapia será minimizarlos $(8,12,13,16,17)$. Grado de recomendación: $D$.

- R.24: muchos de los pacientes que presentan DIO precisan tratamientos coanalgésicos y coadyuvantes adicionales capaces de optimizar la analgesia basal y disminuir su toxicidad $(8,12,13,16,17)$. Grado de recomendación: $D$.

- R.25: el conocimiento de las interacciones entre los fármacos es importante para obtener una mayor eficacia del tratamiento $(7,16)$. Grado de recomendación: $D$.

- R.26: el tratamiento óptimo del dolor neuropático se basa en el uso de fármacos coanalgésicos, fundamentalmente los anticomiciales y/o antidepresivos, que actúan también en la prevención de las crisis de DIO. En caso de aparición de este se recomienda -sin ser la opción terapéutica ideal- la administración de opioides de acción inmediata* $(1,2,7,16)$. Grado de recomendación: $D$.

- R.27: la vía de administración del tratamiento debe adecuarse a la situación clínica y psicosocial del paciente, así como a su ubicación (si permanece hospitalizado, si es ambulatorio o si recibe asistencia domiciliaria) (16). Grado de recomendación: D.

- R.28: según la OMS, los opioides mayores son el tratamiento recomendado para el DIO, pero es imprescindible que el paciente esté recibiendo también opioides para el tratamiento del dolor basal (tolerancia) (18). Grado de recomendación: $D$.

- R.29: deben utilizarse analgésicos potentes con rápido inicio de acción y con un mínimo metabolismo de primer paso hepático, como son los fármacos administrados por vía transmucosa, i.v., s.c. o espinal, ya que aseguran una elevada biodisponibilidad $(7,8,18)$. Grado de recomendación: $D$.

- R.30: el fármaco ideal para el tratamiento del DIO deberá cumplir las siguientes especificaciones $(7,8,18)$ :

- Ser un analgésico potente.

- Tener un inicio de acción rápido (10 minutos o menos).

- Tener una corta duración del efecto (2 horas o menos).

- Poseer mínimos efectos secundarios.

- Ser de fácil administración (cómodo, no invasivo y autoadministrable).

Grado de recomendación: $D$

- R.31: el fentanilo, al presentar una elevada potencia analgésica y alta lipofilia, es el principio activo que más se ajusta a las necesidades analgésicas del dolor

*Dicha recomendación se ha reformulado para adquirir mayor consistencia. irruptivo en el momento actual, con independencia del opioide mayor utilizado para el control del dolor basal $(7,8)$. Grado de recomendación: $D$.

- R.32: a continuación se detallan las características generales de los opioides de liberación rápida (SAO) e inmediata (ROO) (19) (Tabla III). Grado de recomendación: $D$.

- R.33: los grupos de fármacos denominados SAO y ROO pueden ser útiles para el tratamiento del DIO predecible, ya que permiten su administración con anterioridad a la aparición del dolor ocasionado por una maniobra realizada por el propio paciente, su cuidador o el personal sanitario $(18,20,21)$. Grado de recomendación: $D$.

- R.34: la morfina de acción rápida no es, habitualmente, el medicamento óptimo para el tratamiento del DIO debido a su lento inicio de acción y a la larga duración del efecto analgésico; su papel se limita a la prevención del dolor incidental volitivo o procedimental (18,21). Grado de recomendación: D.

- R.35: los ROO (fentanilo de liberación inmediata) son los fármacos de elección para el tratamiento del DIO debido a sus características farmacocinéticas y al rápido inicio de acción, muy especialmente en el dolor idiopático o espontáneo (18). La selección de una u otra presentación y vía de utilización (oral, sublingual o nasal) dependerá, especialmente, de la situación clínica y las preferencias del paciente. Grado de recomendación: $D$.

- R.36: hay que titular siempre la dosis del fármaco usado para el DIO (igual que se titula para el dolor basal), ya que no se dispone de una equivalencia entre la dosis de LAO y de ROO. Se recomienda empezar con la menor dosis disponible del fármaco e ir escalando dosis a intervalos horarios hasta encontrar la mínima dosis eficaz (18) (Fig. 3). Grado de recomendación: D.

- R.37: el dolor es un síntoma cambiante y evolutivo y por ello su tratamiento debe ser dinámico. Es preciso realizar un seguimiento del paciente durante la fase de titulación del fármaco, cuyo objetivo es evaluar la eficacia y la tolerabilidad del tratamiento analgésico, así como conocer cualquier cambio en la naturaleza del DIO. Se recomienda una primera evaluación antes de trascurridas $72 \mathrm{~h}$ desde el inicio del tratamiento; en caso de considerarse necesario, se debe modificar la dosis hasta lograr el adecuado control del dolor. Es conveniente que el paciente anote en su hoja de prescripciones -tanto a nivel intrahospitalario como extrahospitalario- la dosis, el intervalo y el número máximo posible de dosis diarias del fármaco administradas $(8,13,18)$. Grado de recomendación: $D$.

- R.38: una parte del éxito del tratamiento del DIO se basa en la educación sanitaria que se logre trasmitir al paciente y a su entorno acerca del dolor y su mane- 
TABLA III. CARACTERÍSTICAS GENERALES DE LOS OPIOIDES DE LIBERACIÓN RÁPIDA(SAO) E INMEDIATA (ROO). ADAPTACIÓN DE FISHER ET AL. (19)

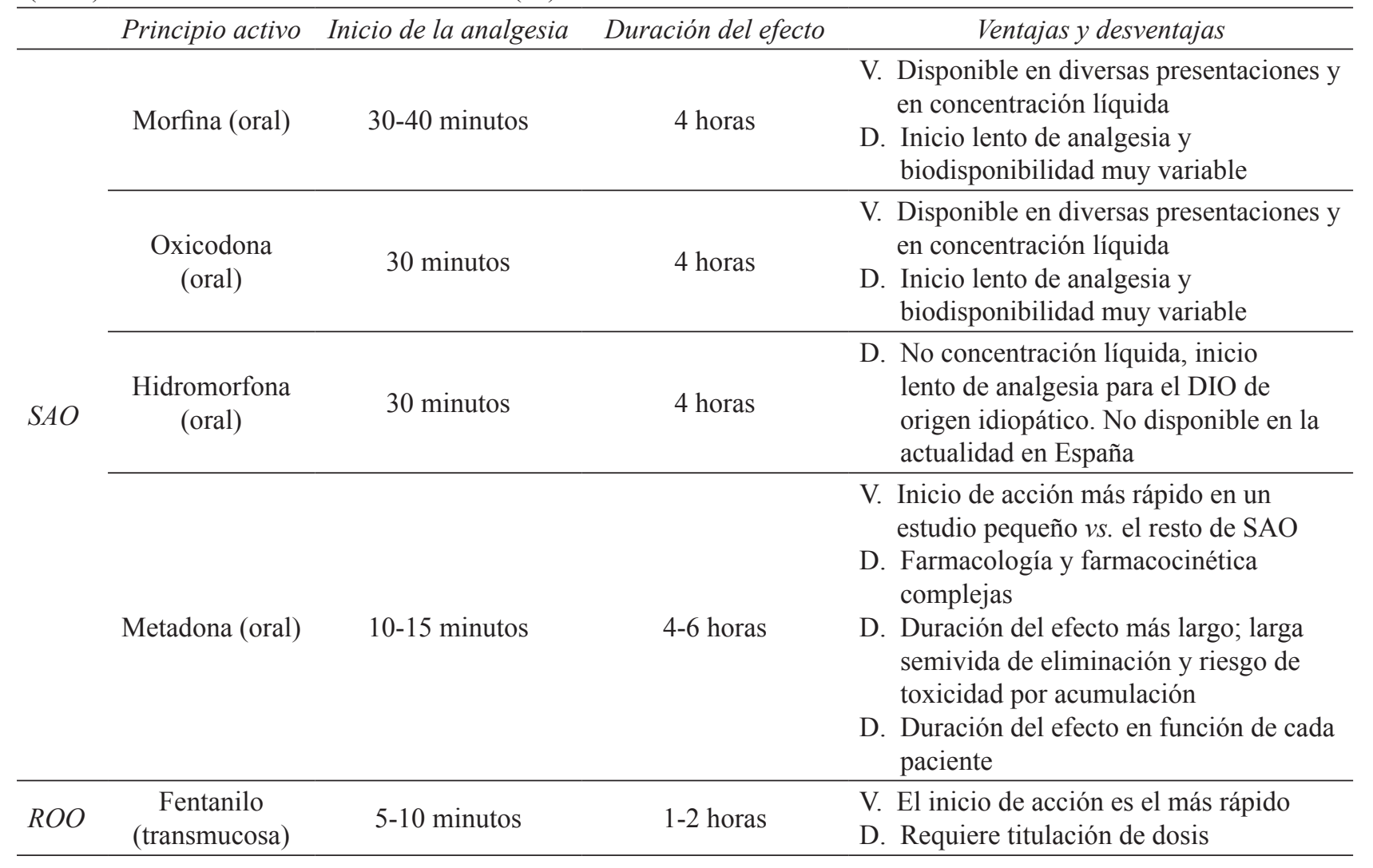

Tiempo X según ficha técnica fármaco $(F)$

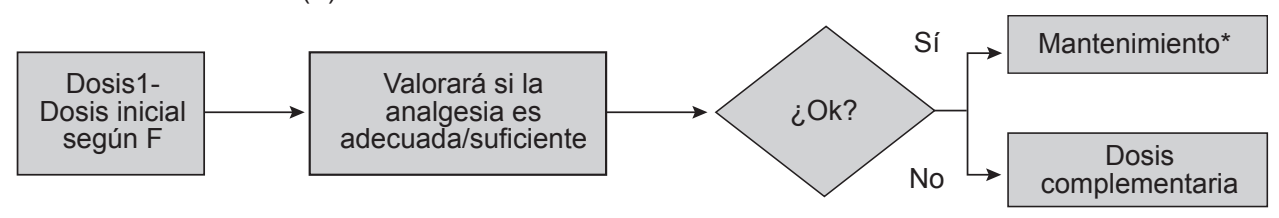

La dosis utilizada (D1) queda establecida como dosis para el paciente para tratar los siguientes episodios, siempre que siga siendo efectiva. En caso de no ser efectiva, valorar el escalado de dosis.

Se puede suministrar una dosis complementaria, según ficha técnica del fármaco. La suma (D1 + dosis complementaria) queda establecida como dosis de inicio para el siguiente episodio de DIO.

No se debe suministrar fármaco para tratar el siguiente episodio antes del tiempo que se indique en la ficha técnica.

Fig. 3. Proceso de titulación de un fármaco para el DIO.

jo, puesto que mejora el cumplimiento terapéutico y minimiza o previene los posibles efectos adversos. El especialista debe destacar la importancia de la administración precoz del tratamiento durante el episodio $(8,13,18)$. Grado de recomendación: D.
- R.39: a pesar de que el dolor basal esté bien controlado, la presencia de DIO es un motivo de insatisfacción terapéutica global para el paciente y condiciona en gran medida su calidad de vida $(8,13,18)$. Grado de recomendación: $D$. 
- R.40: la estrategia terapéutica del DIO (incluidas la coanalgesia, la radioterapia, la rehabilitación, etc.) debe constar en la historia clínica y en el informe del paciente $(8,13,18)$. Grado de recomendación: $D$.

- R.41: el seguimiento telefónico del paciente con DIO, cuando se dispone de medios para realizarlo, es una estrategia eficaz para el control evolutivo. Grado de recomendación: $\sqrt{ }$.

- R.42: los equipos de Atención Primaria deben implicarse en el tratamiento y seguimiento del paciente con DIO. Grado de recomendación: $\sqrt{ }$.

\section{CONCLUSIONES}

Este documento ha sido elaborado con la intención de facilitar la toma de decisiones en la práctica clínica de los médicos implicados en el manejo del paciente oncológico, sea cual sea su especialidad.

Entre las afirmaciones y recomendaciones que han surgido destacan las siguientes:

- Es importante aclarar y homogeneizar la definición de DIO, de forma que se establezca un concepto común y desaparezca la confusión actual.

- El DIO tiene suficiente relevancia clínica como para que se conozca su verdadera prevalencia, la frecuencia de aparición de sus distintos tipos y su relación con el estadio tumoral o con los diferentes tipos y localizaciones tumorales.

- El diagnóstico del DIO debe ser específico e incluir una anamnesis dirigida, una exploración física y las pruebas complementarias que fueran precisas.

- El DIO debe tratarse de forma idónea, para lo cual se recomienda el uso de fármacos potentes con rápido inicio de acción y corta duración. En la actualidad, el principio activo óptimo es el fentanilo, ya que es el que más se ajusta a las necesidades analgésicas del dolor irruptivo y la vía más rápida y cómoda es la transmucosa, ya sea oral o nasal.

- Este tratamiento debe ser siempre complementario del tratamiento opioide usado para el dolor basal y la dosis debe ser titulada, pues no existe equianalgesia entre los opioides de tipo LAO o ROO y los fentanilos de acción inmediata.

- La reevaluación precoz se considera esencial en el proceso de titulación y los efectos adversos deben prevenirse o, al menos, minimizarse, tal y como se hace en el tratamiento del dolor basal.

- No debe olvidarse la existencia de fármacos y técnicas coanalgésicas y coadyuvantes que pueden mejorar el control del dolor y disminuir la dosis de opioides.

Con el fin de facilitar la implementación del documento, el grupo cree necesario que se realice una difusión del mismo y que sea un documento dinámico, es decir, sujeto a las revisiones y a los cambios que puedan extraerse tanto de la práctica clínica como de los avances surgidos de la investigación en dolor. Por ello, creemos necesario que, tras un periodo de implantación, se establezca un grupo de trabajo que permita evaluar la adecuación del documento y formular las modificaciones oportunas, si fueran precisas.

\section{AGRADECIMIENTOS}

Agradecemos a Cephalon Pharma, Ferrer y Teva Pharma haber ofrecido una beca sin restricciones para la elaboración del trabajo, y a Sonia Pisa y Andreu Covas, de Gestió Organització Comunciació, S.A., la conducción de la metodología utilizada para elaborar el documento de consenso.

CORRESPONDENCIA:

Yolanda Escobar Álvarez

Hospital Universitario Gregorio Marañón

C/ Doctor Esquerdo, 46

28007 Madrid

e-mail: yolandaesco@yahoo.es

\section{BIBLIOGRAFÍA}

1. Caraceni A, Martini C, Zecca E, et al. Breakthrough pain characteristics and syndromes in patients with cancer pain. An international survey. Palliat Med 2004;18(3):177-83.

2. Portenoy RK, Payne D, Jacobsen P. Breakthrough pain: characteristics and impact in patients with cancer pain. Pain 1999;81(1-2):129-34.

3. Porta-Sales J, Gómez-Batiste X, Tuca-Rodríguez A, MadridJuan F, Espinosa-Rojas J, Trelis Navarro J. WHO analgesic ladder - or lift? Eur J Palliat Care 2003;10:105-9.

4. Mercadante S, Zagonel V, Breda E, Arcara C, Gebbia V, Porzio $\mathrm{G}$, et al. Breakthrough pain in oncology: A longitudinal study. J Pain Symptom Manage 2010;40(2):183-90.

5. Fortner BV, Okon TA, Portenoy RK. A survey of pain-related hospitalizations, emergency department visits, and physician office visits reported by cancer patients with and without history of breakthrough pain. J Pain 2002;3(1):38-44.

6. Abernethy AP, Wheeler JL, Fortner BV. A health economic model of breakthrough pain. Am J Manag Care 2008;14(5 Supl. 1):S129-140.

7. Porta-Sales J, Garzón Rodríguez C, Julia Torras J, Casals Merchan M. Cancer-related breakthrough pain. Med Clin (Barc) 2010;135(6):280-5.

8. Zeppetella G. Succesful Management of Breakthrough Cancer Pain. London: Evolving Medicine Ltd.; 2010.

9. UNICEF. Visualisation in participatory programmes. A manual for facilitators and trainers involved in participatory group events. Available at: http://portals.wi.wur.nl/files/docs/ ppme/VIPP_Unicef.pdf, 2011.

10. Scottish Intercollegiate Guidelines Network. SIGN 50: A guideline developer's handbook. Edinburgh; 2008. 
11. Portenoy RK, Hagen NA. Breakthrough pain: Definition, prevalence and characteristics. Pain 1990;41(3):273-81.

12. Mercadante S, Radbruch L, Caraceni A, Cherny N, Kaasa S, Nauck F, et al. Episodic (breakthrough) pain: Consensus conference of an expert working group of the European Association for Palliative Care. Cancer. 2002;94(3):832-9.

13. Davies AN, Dickman A, Reid C, Stevens AM, Zeppetella $\mathrm{G}$. The management of cancer-related breakthrough pain: Recommendations of a task group of the Science Committee of the Association for Palliative Medicine of Great Britain and Ireland. Eur J Pain 2009;13(4):331-8.

14. Portenoy RK, Forbes K, Lussier D, Hangs G. Difficult pain problems: An integrated approach. In: Doyle D, Hanks G, Cherny N, Calmen K, editors. Oxford Textbook of Palliative Medicine. $3^{\mathrm{a}}$ ed. Oxford: Oxford University Press; 2004. p. 438-58.

15. Laverty DM, Davies A. Assessment. In: Davies A, editor. Cancer-related breakthrough pain. Oxford: Oxford University Press; 2006. p. 23-30.
16. Bennett D, Burton AW, Fishman S, et al. Consensus Panel Recommendations for the Assessment and Management of Breakthrough Pain PART 1 ASSESSMENT. P\&T ${ }^{\circledR}$ 2005;30(5):296-301.

17. Mercadante S. The use of rapid onset opioids for breakthrough cancer pain: The challenge of its dosing. Crit RevOncol/ Hematol 2011;80(3):460-5.

18. Bennett D, Burton AW, Fishman S, et al. Consensus Panel Recommendations for the Assessment and Management of Breakthrough Pain PART 2 MANAGEMENT. P\&T ${ }^{\circledR}$ 2005;30(6):354-61.

19. Fisher K, Stiles C, Hagen NA. Characterization of the early pharmacodynamic profile of oral methadone for cancer-related breakthrough pain: A pilot study. J Pain Symptom Manage 2004;28(6):619-25.

20. Kleeberg UR, Filbet M, Zeppetella G. Fentanyl buccal tablet for breakthrough cancer pain: Why titrate? Pain Pract 2011;11(2):185-90.

21. CADIME. Tratamiento del dolor irruptivo. Bol Ter Andal 2011:27(4). 